\title{
Preconcepciones y actitudes
} del profesorado de Magisterio ante la incorporación en su docencia de competencias para la sostenibilidad ${ }^{1}$

\section{Preconcepcions and attitudes of teachers of Teaching School about incorporated sustainability competences in their teaching}

\author{
M. Ángeles Ull, Albert Piñero, M. Pilar Martínez-Agut, Pilar Aznar Minguet \\ Universidad de Valencia \\ angels.ull@uv.es, albert.pinero@uv.es,mdelpi@uv.es, pilar.aznar@uv.es
}

RESUMEN - Se ha realizado un estudio empírico con una muestra de profesores de Magisterio de la Universidad de Valencia para analizar sus preconcepciones y actitudes respecto a la incorporación de la sostenibilidad en la docencia de sus asignaturas. La metodología utilizada conforma una estructura descriptivo-exploratoria y evaluativa basada en un diseńo de encuesta. Los datos objetivos nos permiten conocer la situación del profesorado en relación a la introducción de la sostenibilidad en las titulaciones de Maestro de Educación Infantil y Maestro de Educación Primaria, y el desarrollo de competencias para la sostenibilidad pertinentes.

PALABRAS CLAVE: competencias; sostenibilidad; profesorado; Educación Infantil; Educación Primaria.

ABSTRACT • An empirical study has been done with a sample of teachers of Teaching School in the University of Valencia to analyze these teachers' ideas and attitudes about introducing sustainability in their subjects. A descriptive-exploratory methodology based on a survey design was used. The objective data enable us to know the situation of teachers in relation to introducing sustainability in Early Childhood Education Teacher Degree and Primary Education Teacher Degree developing relevant competences for sustainability

KEYWORDS: competencies; sustainability; teachers; Early Childhood Education; Primary Education.

Fecha de recepción: marzo 2012 • Aceptado: enero 2013

1. Este artículo forma parte de la investigación financiada por el MICINN: Proyecto SEJ 2007-67063/EDUC: "Ambientalización curricular: diseño y análisis de intervenciones de Diálogo Disciplinar para el desarrollo de competencias sostenibilizadoras básicas en la educación universitaria (Titulaciones de Educación Infantil y Primaria)». Investigadora principal: Pilar Aznar Minguet. 


\section{INTRODUCCIÓN}

Al concluir el primer decenio del siglo XXI, el mundo afronta retos y problemas importantes, complejos e interrelacionados, que atañen al medio ambiente, el desarrollo humano y a los estilos de vida; los problemas están vinculados entre sí y su solución exige compromisos políticos más enérgicos y acciones más decididas. Los desafíos provienen de valores que han creado sociedades insostenibles, tema que ha sido analizado por muchos autores, entre ellos Elizalde (2009), Vilches y Gil (2009) o Morin (2011).

Este nuevo marco social, producto de los importantes cambios operados durante los últimos años, ha originado una necesaria búsqueda de nuevos enfoques en los procesos de aprendizaje que, unida a los nuevos criterios de Convergencia Europea en el Espacio Europeo de Educación Superior, torna esta coyuntura de los primeros años del siglo XXI en un escenario óptimo para la introducción del paradigma de la sostenibilidad en todos los procesos de formación, tal y como recomienda la UNESCO, y para lo cual esta instauró la Década de la Educación para el Desarrollo Sostenible (2005-2014). La universidad actual tiene que ser una institución comprometida con los principios del desarrollo sostenible, lo cual exige una profunda revisión en sus actividades curriculares, sus tareas de gestión y sus trabajos de investigación (Toledo, 1999; Sterling, 2005; Aznar y Ull, 2009).

El presente estudio trata de especificar competencias básicas y adoptar criterios de sostenibilidad para reformular y orientar los programas curriculares y aplicarlos en las diferentes prácticas formativas; desde esta perspectiva una educación para el desarrollo sostenible en el siglo XXI puede ayudar a la sociedad a hacer frente a los diferentes problemas; prioritariamente los relativos al agua, la energía, el cambio climático, la atenuación del riesgo y los desastres, la pérdida de la biodiversidad, la crisis alimentaria, las amenazas contra la salud, la vulnerabilidad social o la inseguridad. Pero queda mucha tarea por delante para desarrollar procesos de formación que propicien la capacidad de pensar superando las fronteras de la especialización profesional y académica (Orr, 2010).

La Educación para el Desarrollo Sostenible (EDS) es esencial para el surgimiento de nuevas ideas sobre la economía, la sociedad o el medio ambiente y contribuye a crear sociedades resistentes, saludables y sostenibles, mediante un enfoque sistémico e integrado; además, confiere nueva pertinencia, calidad, significado y finalidad a los sistemas de enseñanza y formación. Tal y como indica Sauvé (2010), es necesario formar a ciudadanos capaces de realizar opciones responsables, individuales y colectivas, en materia de transporte, consumo, alimentación, salud, etc.

La finalidad general es formar a todos los estudiantes universitarios, independientemente de la titulación que estudien, en competencias básicas para la sostenibilidad, de manera que una vez egresados, cuando ocupen sus puestos de trabajo, puedan tomar decisiones y realizar acciones profesionales (y personales) desde criterios sostenibles.

La introducción de competencias para la sostenibilidad en el currículum (Barth et al., 2007) puede ser una potente herramienta para que la formación del alumnado universitario contemple los contenidos necesarios para capacitarles en el desarrollo de sus actividades profesionales desde criterios éticos de responsabilidad hacia el desarrollo sostenible. La sostenibilidad implica, tal y como recoge Geli (2002), un gran abanico de conocimientos y habilidades para la acción que sobrepasan la parcelación del saber requiriendo además un tratamiento didáctico desde criterios éticos de responsabilidad, lo que hace necesario la inclusión de la eco-ética en la educación del futuro.

Se necesita un tipo de educación que permita a los estudiantes vivir de manera sostenible, competente y digna, reconociendo su dependencia del entramado de la vida. Esto requiere cambios fundamentales en el currículum, así como una visión más amplia del papel que desempeñan las instituciones educativas, ya que tal y como indica David Orr: «las ideas que sirven de fundamento para la educación superior moderna en todo el mundo reflejan un mundo que dejó de existir hace mucho tiempo» (2010: 156). 


\section{Orientaciones internacionales sobre la introducción de la sostenibilidad en la docencia}

La Declaración de la Década de la Educación para el Desarrollo Sostenible (DEDS) (2005-2014) se dirige a posibilitar la construcción de un mundo en el que todos tengan igualdad de acceso a la educación a través de la cual aprender los valores, comportamientos y estilos de vida coherentes para un futuro sostenible y para la transformación positiva de la sociedad. Estas cuestiones aparecen reflejadas como prioridades en la planificación de los programas y actividades que hay que desarrollar para conseguir los objetivos de la Década de la EDS, tales como: reducción de la pobreza, igualdad de sexos, promoción de la salud, protección del medio ambiente, transformación rural, derechos humanos, comprensión cultural y paz, producción y consumo responsables, respeto a la diversidad cultural o acceso igualitario a las TIC (UNESCO, 2005a). Con la declaración de la Década, Naciones Unidas ofrece una gran oportunidad para consolidar los procesos iniciales de «buenas prácticas» en la Comunidad Internacional de los estudios superiores, reorientar las funciones de enseñanza e investigación, generar respuestas creativas a los problemas ambientales y educar para la sostenibilidad.

\section{REFERENTES BÁSICOS EN LA INTRODUCCIÓN DE LA SOSTENIBILIDAD EN LA FORMACIÓN UNIVERSITARIA}

La Universidad y el conjunto de instituciones de enseñanza superior juegan un papel clave en este proceso, por ser instituciones creadoras de opinión y generadoras de los paradigmas metodológicos que rigen el progreso económico y social; y porque la sociedad requiere capital humano capaz de enfrentarse al reto de la sostenibilidad (Aznar Minguet, 2006). La formación universitaria no puede limitarse a facilitar el desarrollo de capacidades científicas y técnicas; es necesario también facilitar el desarrollo de competencias, valores, comportamientos y modos de vida coherentes con un futuro viable.

Aunque realmente en el ámbito universitario la educación para la sostenibilidad se ha iniciado en la década de los noventa, existen varias referencias a nivel internacional de la coordinación de las universidades interesadas en la introducción de la sostenibilidad que ya se han citado ampliamente en otros artículos (Ull et al., 2010a; Junyent et al., 2011; Aznar et al., 2011; Vega y Álvarez, 2011).

A título de ejemplo cabe mencionar las Conferencias Internacionales sobre Educación Superior para el Desarrollo Sostenible: en 2005 tuvo lugar la I Conferencia en la Universidad de Lüneburg, en la que más de 150 expertos debatieron sobre los nuevos retos de la educación superior para el desarrollo sostenible desde una perspectiva global; desde esta Conferencia se propulsó el desarrollo de la Agenda 21 Universitaria. En 2007 se realizó la II Conferencia en San Luis de Potosí (Méjico), como una contribución significativa a la Década de Naciones Unidas de la educación para el desarrollo sostenible; en esta conferencia se centraron los debates en las posibles contribuciones de las universidades a los procesos de cambio (locales, regionales e internacionales) durante la transición a la sostenibilidad global, poniendo especial énfasis en la definición, reorientación y medición de las competencias para el cambio global sostenible, el desarrollo de redes globales y sociedades locales, así como la promoción de cambios concretos en los estilos de vida académica a nivel de gestión, de docencia, de investigación y de participación de la comunidad universitaria hacia el desarrollo sostenible. En el 2009 se celebró en Bonn la III Conferencia, en la que se plantearon, entre otros objetivos:

- Apoyar la incorporación de temas del desarrollo sostenible, mediante una estrategia integrada y sistémica, en todos los niveles de la educación formal, no formal e informal, en particular mediante la elaboración de métodos pedagógicos eficaces, la formación de docentes, las prácticas de enseñanza, los planes y programas de estudio, los materiales didácticos y el fomento del liderazgo en el sector educativo, y también mediante el reconocimiento de la significativa 
contribución de la educación no formal y el aprendizaje informal, la formación profesional y el aprendizaje en el lugar de trabajo. El desarrollo sostenible es un tema transversal importante para todas las disciplinas y todos los sectores.

- Reorientar los programas de elaboración de planes de estudio y formación de docentes, con miras a integrar la EDS en los programas de formación inicial y de perfeccionamiento para docentes en funciones. Alentar a las instituciones de formación de docentes, los maestros y los profesores a crear redes y a elaborar e investigar las prácticas pedagógicas idóneas. En particular, ayudar a los docentes a formular estrategias de EDS aplicables a clases numerosas y a evaluar los procesos de aprendizaje de la EDS.

Todos estos documentos coinciden en señalar que la educación superior es una herramienta clave para alcanzar el Desarrollo Sostenible, lo cual supone que la Universidad debe formar a profesionales capaces de utilizar sus conocimientos, no solo en un contexto científico, sino también para dar respuesta a las necesidades sociales y ambientales. Los profesionales que la Universidad forma deben:

- comprender cómo su actividad profesional interactúa con la sociedad y el medio ambiente, local y globalmente, para identificar posibles desafíos, riesgos e impactos;

- trabajar en equipos multidisciplinares, para dar solución a las demandas impuestas por los problemas socio-ambientales, incluyendo propuestas de alternativas profesionales que contribuyan al desarrollo sostenible;

- aplicar un enfoque holístico y sistémico a la resolución de los problemas profesionales;

- participar activamente en la discusión, definición y evaluación de políticas y acciones, tanto en el ámbito público como privado, para contribuir a redirigir la sociedad hacia un desarrollo más sostenible;

- aplicar los conocimientos profesionales de acuerdo con principios deontológicos y principios éticos relacionados con los valores de la sostenibilidad;

- comprender la contribución de su trabajo en los diferentes contextos profesionales, culturales, políticos y sociales en los que interactúan, y su influencia en el desarrollo de una mayor conciencia de la necesidad de sostenibilidad.

En el ámbito español existen dos referentes básicos que hay que destacar: la red ACES² (Red de Ambientalización Curricular ${ }^{3}$ de los Estudios Superiores) y el grupo de trabajo de Sostenibilización curricular de la CADEP-CRUE. ${ }^{4}$

En el año 2000 se constituyó la Red ACES, dentro del Programa Alfa de la Unión Europea. Dicha Red está constituida por 5 universidades europeas y 6 de América Latina. La red ACES tiene objetivos confluyentes en cada una de sus universidades siendo coherentes con los objetivos que nos proponemos en este estudio para la Universidad de Valencia.

En 2009 se constituyó dentro de la CADEP (Comisión Sectorial de Calidad Ambiental, Desarrollo Sostenible y Prevención de Riesgos), de la CRUE, el Grupo de trabajo sobre Sostenibilización curricular, al que pertenecen por el momento 13 universidades. El grupo tiene como objetivo, entre otros, la inclusión de criterios y competencias para la sostenibilidad en todos los planes de estudio universitarios y es heredero del grupo de universidades que venía reuniéndose desde varios años antes (Barrón et al., 2010).

2. Red ACES: http://insma.udg.es/ambientalitzacio/web_alfastinas/angles/a_index.htm.

3. Más recientemente se ha hablado de sostenibilización curricular, aunque hoy en día se prefiere el uso de introducción de la sostenibilidad en el currículum o en los planes de estudio, por ser más claro; en todos los casos se está hablando de lo mismo y en este texto lo utilizamos indistintamente.

4. CRUE: http://www.crue.org/Sostenibilidad/. 


\section{Directrices sobre introducción de la sostenibilidad en los planes de estudio (CRUE)}

Las Directrices para la introducción de la sostenibilidad en los currículos, documento aprobado por el Grupo de trabajo para la Calidad Ambiental y el Desarrollo Sostenible de la Conferencia de Rectores de las Universidades Españolas (CRUE) en 2005, fue reasumido y potenciado en la reunión de la Comisión Sectorial de Calidad Ambiental, Desarrollo Sostenible y Prevención de Riesgos (CADEP) de Granada en 2009, y finalmente aprobada en Asamblea General de la CADEP en marzo de 2012. Cabe destacar las siguientes conclusiones:

- Promover desde la Comisión de Calidad Ambiental, Desarrollo Sostenible y Prevención de Riesgos de la CRUE, que la Comisión Sectorial de Estudios u Ordenación Académica de la CRUE asuma la promoción de la sostenibilidad en todos los planes de estudio, según lo establecido en el RD 1393/2007 y las Directrices anteriormente citadas.

- Necesidad de incluir la sostenibilidad en los planes de estudio a través de: Transversalidad en las asignaturas de los currículos, desarrollando las competencias generales de sostenibilidad; Introducir asignaturas obligatorias transversales a todas las titulaciones donde se desarrollen las competencias relacionadas con la sostenibilidad; Introducir asignaturas optativas en todas las titulaciones donde se desarrollen las competencias relacionadas con la sostenibilidad; Desarrollo de proyectos interdisciplinares sobre problemáticas socio-ambientales.

- Reconocer la potencialidad de todas las áreas para crear espacios de reflexión e impulsar el cambio cultural hacia la sostenibilidad.

\section{Normativa sobre introducción de la sostenibilidad en los planes de estudio de los grados de Maestro de Educación Infantil y Maestro de Educación Primaria}

La Ley Orgánica 4/2007 de Universidades se desarrolla con el Real Decreto 1393/2007, por el que se establece la ordenación de las enseñanzas universitarias oficiales. En el preámbulo de dicho R.D. se especifica que «se debe tener en cuenta que la formación en cualquier actividad profesional debe contribuir al conocimiento y desarrollo de los Derechos Humanos, los principios democráticos, los principios de igualdad entre mujeres y hombres, de solidaridad, de protección medioambiental, de accesibilidad universal y diseño para todos, y de fomento de la cultura de la paz».

En aquellos planes de estudio con habilitación profesional, se indican, mediante Resoluciones, las condiciones a las que deberán adecuarse los planes de estudios conducentes a la obtención de títulos que habiliten para el ejercicio de una profesión regulada, entre ellos los títulos de Maestro; se han publicado también las Órdenes para la Verificación y en dichas órdenes se recogen las competencias generales y específicas de esas titulaciones (Martínez-Agut et al., 2007).

Los títulos de grado de Maestro de Educación Infantil y Maestro de Educación Primaria vienen reseñados en la Resolución de 17 de diciembre de 2007 y se ha publicado la Verificación de los títulos universitarios oficiales que habiliten para el ejercicio de la profesión de Maestro de Educación Infantil, mediante la Orden ECI/3854/2007 y para la de Maestro de Educación Primaria, mediante la Orden ECI/3857/2007. En estas órdenes puede verse que, en los títulos de Grado de Maestro se ha establecido la materia "Sociedad, familia y escuela», que en la titulación de Maestro de Educación Infantil, tiene una carga docente de 100 créditos y en la titulación de Maestro de Educación Primaria, tiene una carga docente de 60 créditos, a través de los cuáles, los alumnos tienen que adquirir las competencias de, entre otras: «Analizar e incorporar de forma crítica las cuestiones más relevantes de la sociedad actual... como el desarrollo sostenible». 
Tal y como recoge en su introducción el documento de UNESCO Directrices y recomendaciones encaminadas a reorientar la formación de docentes para abordar el tema de la sostenibilidad: «Las escuelas normales cumplen funciones vitales en la comunidad educativa mundial y tienen la capacidad de suscitar transformaciones en los sistemas educativos que configurarán los conocimientos y competencias de las generaciones futuras. La educación se califica con frecuencia de gran esperanza para crear un futuro más sostenible y los institutos de formación pedagógica serán agentes de cambio fundamentales al transformar la educación y la sociedad para que ese futuro sea posible» (UNESCO, 2005b: 9).

La introducción de la sostenibilidad en el curriculum es una tarea colectiva que requiere ser consensuada y cuya viabilidad depende de la implicación de alumnos y profesores. Los alumnos tienen que percibir la posibilidad de cambio y de modificación de los modelos sociales actuales desde el contexto de su formación universitaria para poder transferir a su entorno y aplicar en su futura práctica profesional las estrategias aprendidas con criterios de sostenibilidad. Los futuros profesionales de mañana, que son nuestros estudiantes de ahora, deben conocer y saber cómo actuar desde el punto de vista técnico y ético ante la situación actual del planeta. Los profesores tienen que asumir su responsabilidad en el modelo que se propone a través de los cambios que puedan introducir en el currículum de la materia o materias que imparten, tal como ya indicaban Pujol (2006) y Miranda y Regí (2006). Y para todo ello, la implicación del profesorado como pieza clave del proceso es, en último término, la que posibilita de manera efectiva la incorporación de la sostenibilidad en el currículo.

El objetivo general de este trabajo es aportar la formación básica para el desarrollo de competencias para la sostenibilidad en los procesos de enseńanza/aprendizaje y la aplicación de criterios para propiciar la sostenibilidad en la docencia de las titulaciones de Educación Infantil y Educación Primaria; es preciso orientar el papel de la educación como difusora de valores, actitudes, comportamientos, costumbres y estilos de vida que fomenten la sostenibilidad; y en esta empresa merece especial atención la formación de los futuros educadores de los niños y adolescentes y su influencia para transformar el modelo cultural hacia la sostenibilidad (Pramling Samuelsson y Kaga, 2010).

El presente trabajo se enmarca dentro de un proyecto de investigación más amplio sobre la introducción de competencias para la sostenibilidad en los planes de estudio de los grados de Maestro en Educación Infantil y Maestro en Educación Primaria. Se ha realizado un seminario de diálogo disciplinar (Bonil et al., 2004), con profesorado de la actual Facultad de Magisterio de la Universitat de Valencia, cuyos resultados se recogen en otra publicación (Aznar et al., 2014) y, previamente al citado seminario y como preparación al mismo, se realizaron sendas encuestas al profesorado y al alumnado de Magisterio. El estudio que aquí se presenta es el resultado de la encuesta al profesorado, que permite conocer el punto de partida para la incorporación en su docencia de competencias para la sostenibilidad.

\section{METODOLOGÍA}

Se ha realizado una encuesta para conocer las preconcepciones y actitudes del profesorado de de las titulaciones de Maestro de Educación Infantil y Maestro de Educación Primaria de la Universidad de Valencia, respecto a la introducción de competencias para la sostenibilidad en los procesos de enseñanza-aprendizaje (Aznar et al., 2009). Está basada en un cuestionario anterior de los autores de esta investigación (Aznar et al., 2007), con la inclusión de referencias al diálogo disciplinar y a criterios de sostenibilidad, adaptando algunos de los ítems utilizados por la UNESCO.

\section{Diseño del cuestionario}

La encuesta, que ha sido sometida a la valoración de jueces para su fiabilidad interna, contiene 31 variables propias del tema estudiado o dependientes, además de 18 variables explicativas o independientes. Las 31 variables relativas al tema se han clasificado en 4 categorías: 
1. Identificación (titulaciones en que imparte docencia, Departamento de adscripción, años de experiencia docente, sexo...)

2. Percepción (percepción de los problemas ambientales, percepción de la formación...)

3. Interpretación (opinión sobre acciones genéricas que hay que realizar, situación en sus asignaturas...).

4. Comportamiento (actitudes ante la introducción de la sostenibilidad...)

Las preguntas del cuestionario recogen las opiniones del profesorado sobre: La problemática socioambiental (P1), formación recibida (P2), actualización de conocimientos (P3), la sostenibilidad en la docencia (P4 y P5), temas y acciones que hay que llevar a cabo para potenciar la cultura de la sostenibilidad (P6 a P11), acciones con los estudiantes (P12 a P19) y cuestiones genéricas sobre la introducción de la sostenibilidad en los currículos (P20 a P26). ${ }^{5}$

Para el diseño del cuestionario se utilizaron Formularios de Word 2001, transformados luego hasta Word 2008 para Mac. La base de datos para grabación ha sido Filemaker Pro. Para asegurar la confiabilidad de los datos se diseńó un programa de entrada de datos con filtros que hacen imposible la comisión de errores lógicos de grabación. Una vez grabadas las respuestas de los profesores, se volcó la matriz de datos en Data Desk 6.0, aplicación con la cual se realizó el análisis estadístico. Por último, la creación de cuadros y gráficos se ha realizado con Excel 2008 para Mac. Para la encuesta anterior arriba citada, se generó un sistema permanente de medición del grado de ambientalización curricular, un nuevo indicador, al que denominamos índice Pi que también se ha aplicado a este cuestionario. ${ }^{6}$

\section{Población y muestra}

La población objeto de estudio ha sido la totalidad del profesorado que imparte docencia en la Escuela de Magisterio «Ausias March» de la Universitat de Valencia, durante el curso 2007-2008, en concreto 116 profesores/as. $^{7}$

Esta escuela tiene 4 departamentos adscritos, que son los de Didáctica de las Matemáticas, Didáctica de la Lengua y la Literatura, Didáctica de las Ciencias Experimentales y Sociales y Didáctica de la Expresión Musical, Plástica y Corporal. De los departamentos no adscritos hay varios que tienen un número importante de profesores/as con gran parte de docencia en Magisterio (Teoría de la Educación, Educación Comparada...).

A todos los/las profesores/as se les hizo llegar la encuesta en formato papel y por correo electrónico, si así lo solicitaron. Un total de 52 profesores/as la han contestado, lo que supone un nivel de error del $10.3 \%,{ }^{8}$ dentro de un nivel de confianza del $95.5 \%$, equivalente a 2 s.

5. Puede consultarse el cuestionario completo en: www.uv.es/acuveg (última consulta, 27-08-2012).

6. Es un constructo algebraico, aplicable a las preguntas cuyos ítems están escalados, cuyo rango es, en todos los casos y para todas las cuestiones, $[0 ; 1]$, independientemente de la longitud de la escala respectiva (Piñero et al., 2006). También se han creado coeficientes de ponderación que cuantifican la importancia atribuida en el conjunto a cada una de las distintas variables escaladas. En este artículo el índice aparece en algunas tablas, pero no se hace más referencia al mismo, aunque sí nos servirá para investigaciones posteriores.

7. En el curso académico 2011-1012 esta escuela ha pasado a ser Facultad de Magisterio.

8. Este error es mayor que los que habitualmente se dan en investigaciones cuantitativas con muestras de grandes colectivos, sin embargo sí se producen en estudios sobre pequeñas poblaciones, requiriendo tan solo ser muy prudentes al establecer las conclusiones. 


\section{RESULTADOS}

\section{Caracterización de la muestra}

Los departamentos más representados en la muestra son los de Didáctica de las Ciencias Experimentales y Sociales (26’\%) y Didáctica de la Expresión Musical, Plástica y Corporal (21,2\%) (tabla 1).

Tabla 1.

Departamentos de adscripción del profesorado

\begin{tabular}{|l|c|c|}
\hline VE2. Departamento de adscripción & no respuestas & $\%$ \\
\hline $\begin{array}{l}\text { Didáctica de las Ciencias Experimentales y } \\
\text { Sociales }\end{array}$ & 14 & 26,9 \\
\hline $\begin{array}{l}\text { Didáctica de la Expresión Musical, Plástica y } \\
\text { Corporal }\end{array}$ & 11 & 21,2 \\
\hline Didáctica de la Lengua y la Literatura & 6 & 11,5 \\
\hline Teoría de la Educación & 5 & 11,5 \\
\hline Didáctica y Organización Escolar & 3 & 9,6 \\
\hline Didáctica de las Matemáticas & 2 & 3,8 \\
Educación Comparada e H. de la Educación & 2 & 3,8 \\
\hline Sociología y Antropología Social & 1 & 1,9 \\
\hline Métodos de Investigación y Diagnóstico en & 1 & 1,9 \\
Educación & 1 & 1,9 \\
\hline Psicología Evolutiva y de la Educación & 52 & 100,0 \\
\hline NC
\end{tabular}

El 76,9\% del profesorado que ha respondido a la encuesta imparte asignaturas de tipo troncal u obligatorio. El 44,2\% imparte optativas. El 32,7\% se ocupa de algún Prácticum, y el 28,8\% de asignaturas de libre elección.

El 59,5\% del profesorado entrevistado es funcionario, siendo la mayoría de entre ellos los titulares de universidad y los titulares de escuela universitaria no doctores, representando cada uno de esos grupos el 19,2\%, seguidos por los titulados de escuela universitaria doctores, que son el 13,5\%. De entre el profesorado entrevistado no funcionario/a el 25,0\%, son asociados.

El 75,0\% del profesorado encuestado se dedica en exclusiva a sus funciones universitarias. El 40,4\% tienen una experiencia docente máxima de un decenio. El 21,2\% llevan entre 11 y 20 años, el 17,3\% de 21 a 30 , y un $21,2 \%$ tiene más de 30 ańos de experiencia docente.

La edad del profesorado que ha dado respuesta a la encuesta es media alta. Solo el 21'2\% es menor de 40 años, el 38,5\% tiene entre 40 y 54, y el 40,4\% tienen edades entre 55 y 69 años.

El profesorado entrevistado se distribuye casi al $50 \%$ entre hombres y mujeres. Encontramos un mayor \% de mujeres con respecto a la media de los PDI de la Universitat de Valencia, en la que la relación es del $39 \%$ de mujeres y el $61 \%$ de hombres.

\section{La problemática socio-ambiental (P1)}

Más de la mitad del profesorado entrevistado (61,5\%) afirma que contribuir al desarrollo sostenible es inherente a una actitud profesional respetuosa con el medio (figura 1). 


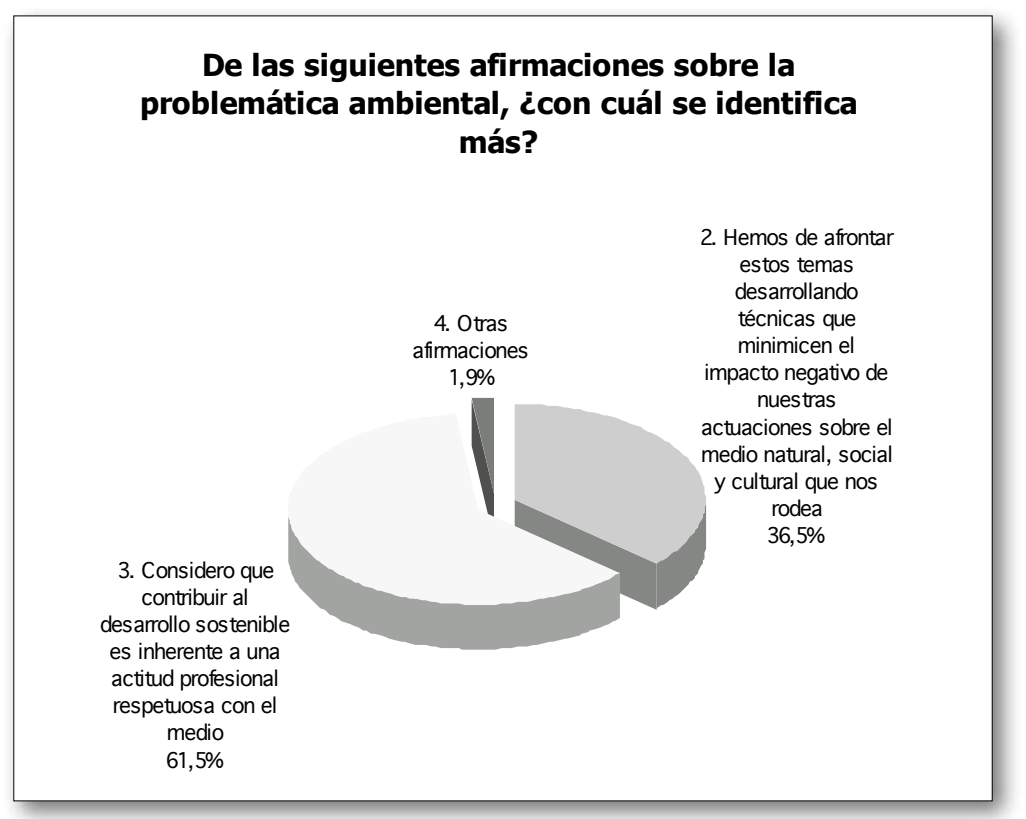

Fig. 1. La problemática socio-ambiental

\section{Formación recibida en la universidad a la luz de la sostenibilidad (P2)}

El 84,6\% de los profesores de Magisterio cree que la formación que han recibido en la universidad apenas les ha preparado para trabajar desde criterios ambientales y de sostenibilidad en el ejercicio de su profesión o actividad. Casi la mitad $(48,1 \%)$ de profesores encuestados confiesan no haber recibido en la universidad preparación alguna para trabajar en el ejercicio de sus funciones docentes e investigadoras desde criterios ambientales y de sostenibilidad, y el 36,5\% afirma que fue exigua. Hay profesores que mantienen haber recibido una formación suficiente $(9,6 \%)$ y hasta completa $(5,8 \%)$.

\section{Actualización de conocimientos (P3)}

La relativamente reciente preocupación por las cuestiones socio-ambientales y su introducción en las instituciones de la enseńanza superior ha llevado a los profesores que no recibieron formación sobre estos temas en la etapa de sus estudios universitarios a ponerse al día en los temas relacionados con la sostenibilidad. Esta formación ulterior la ha realizado el 59,1\%, consultando bibliografía. El 31,6\% dice haberla realizado participando en cursos, jornadas y/o seminarios; un 31,6\% dice haber investigado sobre el tema. Finalmente, el 42,4\% manifiesta haberse actualizado de otras maneras diversas. Hay dos puntos importantes que destacar. El primero, que hay profesores que han realizado varias de las actividades anteriores para ponerse al día, lo que puede suponer un nivel de actualización más que considerable. La segunda, que poco más de una cuarta parte del profesorado encuestado, un $27,5 \%$, no se ha puesto al día en temas relacionados con la sostenibilidad.

\section{La sostenibilidad en la docencia (P4 y P5)}

Tal y como se ha indicado en la introducción, la UNESCO reconoce que es preciso un esfuerzo para introducir contenidos y enfoques relacionados con el medio ambiente y el desarrollo sostenible en las asignaturas de los planes de estudio universitarios, lo que se ha entendido por ambientalización curricular. 
La introducción de contenidos, criterios o enfoques relacionados con la sostenibilidad en los planes de estudios de las instituciones de educación superior, aunque constituye uno de los principios de la Declaración de Bolonia, que han sido traducidos y adaptados por la Conferencia de Rectores de las Universidades espańolas, está siendo un proceso, además de reciente, innovador, aunque lento y complejo. Los profesores constituyen la razón estratégica para su aplicación a nivel de docencia e investigación. Su postura respecto a las posibilidades de introducir criterios relacionados con la sostenibilidad en su ámbito de docencia e investigación es básica para la introducción de la sostenibilidad en el currículum, es por ello que se han realizado varias preguntas sobre este punto.

\section{Introducción de contenidos y enfoques relacionados con la sostenibilidad}

El 86,5\% del profesorado está de acuerdo y es destacable que el 53,8\% dice que ya lo está haciendo (figura 2).

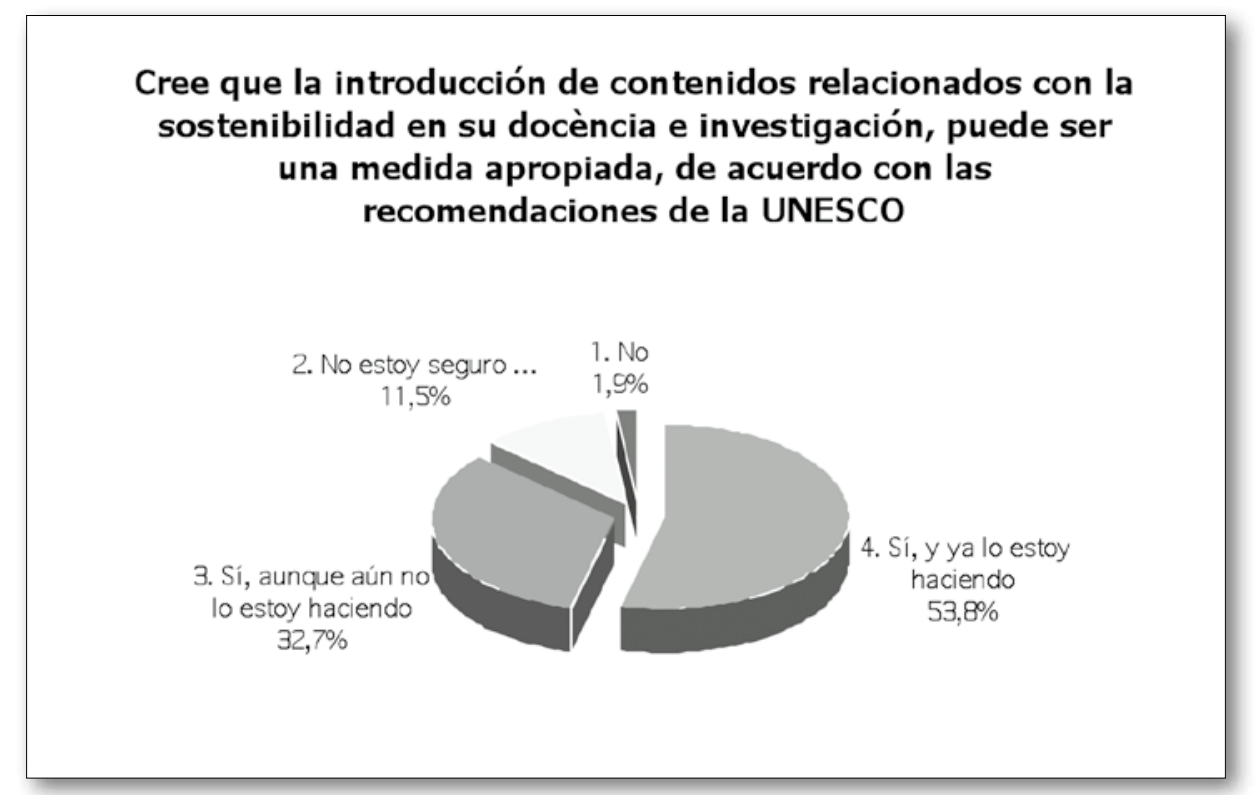

Fig. 2. Introducción de contenidos

\section{Introducción de contenidos y enfoques relacionados con la sostenibilidad en la titulación}

Cuando se les pregunta en concreto por sus asignaturas de las titulaciones de Maestro, la mayoría del profesorado $(88,5 \%)$ cree que es factible introducir contenidos y enfoques relacionados con la sostenibilidad en las asignaturas que imparten o coordinan en las titulaciones de Maestro, frente a un 11,5\% que considera que no es factible. Se ha realizado un cruce relacionando el departamento de adscripción con los profesores que han dado respuesta negativa a la pregunta anterior y se ha concluido que estos pertenecían a departamentos que tienen en común el ser los menos involucrados directamente con la sostenibilidad (Didáctica de las Matemáticas, Didáctica de la Lengua...). 
Temas y acciones que llevar a cabo para potenciar la cultura de la sostenibilidad (P6 a P11)

\section{Temas de la Década de la EDS}

De entre los temas que promociona la UNESCO en la «Década de la Educación para el Desarrollo Sostenible 2005-2014», los profesores encuestados manifiestan tener incorporados como objetivos y contenidos en las asignaturas que imparten en mayor medida: la diversidad cultural $(90,4 \%)$, la comprensión cultural y la paz $(90,4 \%)$, la igualdad de sexos $(86,5 \%)$, los derechos humanos $(80,8 \%)$, la protección del medio ambiente $(73,1)$ y en menor medida el resto de temas (figura 3 ).

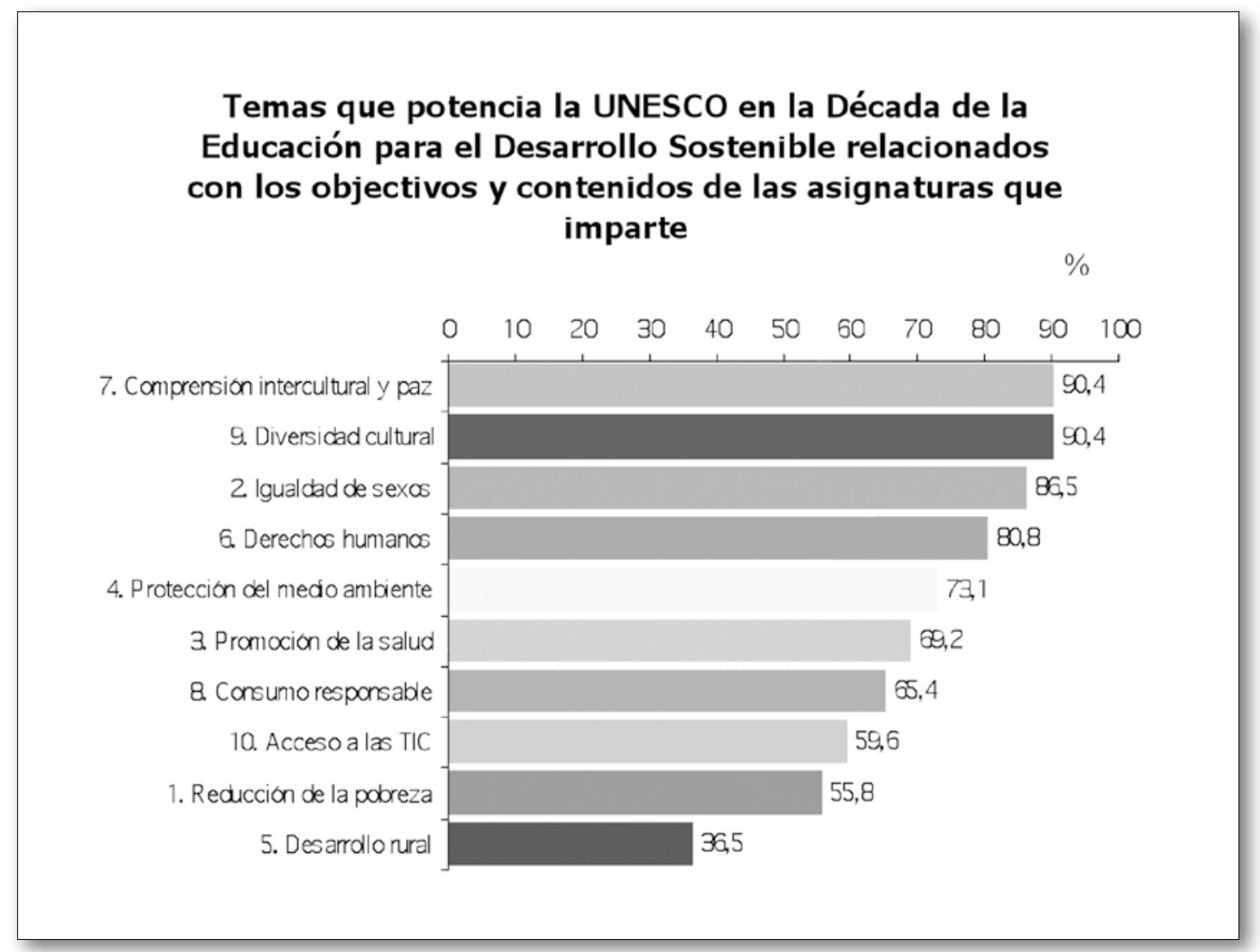

Fig. 3. Temas de la Década de la Educación para el Desarrollo Sostenible 2005-2014

Todos estos temas forman parte de la perspectiva de la sostenibilidad y estas respuestas están en consonancia con el alto porcentaje de profesorado encuestado que ha contestado que ya está introduciendo la sostenibilidad en su docencia.

\section{Inclusión de acciones de formación}

Contemplar la sostenibilidad en los planes de estudio implica la inclusión de acciones específicas de formación (sensibilización, técnicas, habilidades...), que preparen a los futuros graduados de las titulaciones de Magisterio para afrontar los problemas relacionados con la sostenibilidad en el ámbito de sus competencias profesionales como futuros maestros. 
El profesorado encuestado opina mayoritariamente que en los planes de estudios actuales si se incluyen, aunque aún hay mucho por hacer en este campo (67,3\%). Algunos profesores desconocen esta cuestión (13,5\%). Otros abiertamente niegan que se produzca tal inclusión (9,6\%). Por último, algunos otros $(7,7 \%)$ estiman que si se incluyen, aunque a un nivel poco satisfactorio. Puede verse que en un porcentaje alto son muy críticos con lo que queda por hacer en el proceso.

\section{Interacción con otro profesorado}

La interacción entre profesorado que imparte la misma asignatura, así como la interacción con otros/ as profesores/as, es una variable importante que hay que tener en cuenta en el desarrollo de un modelo de formación por competencias; dicha interacción facilita la realización de acciones tendentes a desarrollar las competencias generales y específicas de la titulación que un estudiante tiene que conseguir para graduarse. En el caso del profesorado encuestado el 78,8\% manifiesta tener establecidas dichas interacciones, frente al $21,2 \%$ que declara no tenerlas. Vuelve a ser este un dato muy alentador para la aplicación y desarrollo de las competencias para la sostenibilidad en la docencia de las titulaciones de Magisterio.

\section{Acciones con los estudiantes (P12 a P19)}

\section{Participación en acciones solidarias}

Es muy significativo que un $65,4 \%$ de los/las docentes declaren que, desde las asignaturas que imparten, planifican y/o realizan acciones relacionadas con el desarrollo sostenible, que afectan a la vida personal de los estudiantes y promueven su participación en acciones solidarias.

\section{Trabajo de valores (respeto, tolerancia, equidad...) desde las asignaturas}

Un porcentaje muy elevado de profesorado $(94,2 \%)$ declara que trabaja desde su asignatura los valores de respeto, tolerancia, equidad, etc., que también definen el desarrollo sostenible. Solamente dos afirman que no trabajan estos valores o que no los reconocen como vinculados al desarrollo sostenible. Por tanto, podemos establecer que el profesorado, en general, vincula estos principios con la sostenibilidad.

Esta pregunta cerrada se completa con una pregunta abierta en la que la mayoría del profesorado (35 de 52) hace explícitas las formas de trabajar estos valores que se agrupan a continuación en cuatro categorías de respuesta: temas de metodología, aspectos valorativos, cuestiones de la actualidad y aspectos propios de la materia.

En lo que se refiere a temas de metodología, el profesorado presenta actividades varias: A través de los textos, audio, etc., se hacen reflexiones, discusiones grupales, problemas que se resuelven en el aula, debates, juego de rol, estudio de casos reales o hipotéticos, mesas redondas, etc. (12 respuestas); impregnando los contenidos curriculares con actividades específicas y ejemplos (6 respuestas). Otras respuestas individuales fueron: con la propia actitud docente; con los materiales y recursos utilizados; con el lenguaje; la interacción en el aula es un ejemplo de la aplicación de estos valores; trabajos en equipo, investigación sobre el medio, trabajos de campo. En total, 23 profesores establecen aspectos concretos de trabajo metodológico en el aula, es decir, aspectos propios del trabajo del docente y vinculados con la innovación.

En lo que atañe a respuestas vinculadas con aspectos valorativos: dos docentes vinculan la tradición en la formación de maestros con estos aspectos relacionados con las actitudes y la sostenibilidad, es decir, sería un aspecto externo, que impone la propia vocación del maestro/a. Dos docentes vinculan 
la necesidad social y las condiciones actuales con aspectos relacionados con la sostenibilidad; es decir, sería un aspecto externo, que impone la situación social. Y ocho docentes vinculan su materia con estos aspectos relacionados con la sostenibilidad; es decir, sería un aspecto externo que, en opinión de estos profesores, impone la propia materia.

Así pues, podemos concluir que 23 profesores trabajan los valores vinculados con la sostenibilidad por iniciativa propia y formación continua, y 12 realizan este trabajo por motivaciones externas.

Inclusión de aspectos relacionados con el medio ambiente y con el desarrollo sostenible en las asignaturas que imparten

Ante la pregunta de si actualmente se tratan aspectos relacionados con el medio ambiente y con el desarrollo sostenible en las asignaturas que coordinan y/o imparten, se ofertaban 4 respuestas posibles y había que seleccionar solamente una.

La respuesta de que los tratan, de manera sistemática en todas las asignaturas, la más favorable, la escoge el 28,8\%. Algo más de la mitad del profesorado encuestado, el 53,8\%, dice que incluye de manera esporádica, según la asignatura lo permita, los aspectos relacionados con la sostenibilidad. El $7,7 \%$ dice tratar muy poco esos temas, debido a que sus asignaturas no se prestan a ello, y un $9,6 \%$, dice que no los tratan por ahora.

La pregunta anterior se completó con la reflejada en la figura 4.

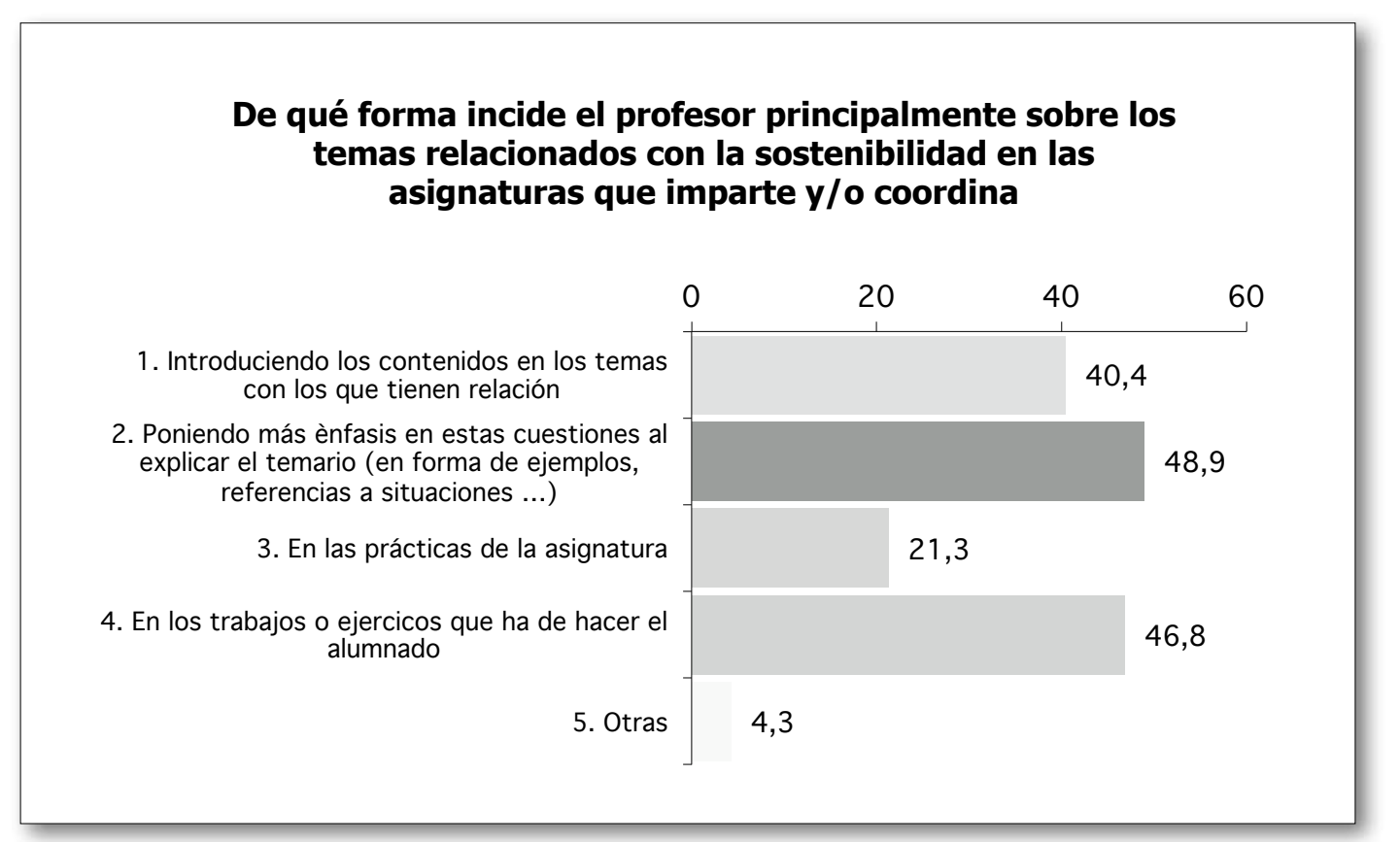

Fig. 4. Incidencia sobre los temas relacionados con la sostenibilidad en las asignaturas

\section{Trasmisión de valores relacionados con la sostenibilidad}

La encuesta incluía una cuestión sobre cuál era, para los profesores, la validez ética de transmitir consciente y positivamente valores en el contexto de sus clases, junto a los saberes que transmiten en su enseñanza (tabla 2). 
Tabla 2.

Trasmisión de valores relacionados con la sostenibilidad

\begin{tabular}{|c|c|c|}
\hline $\begin{array}{l}\text { P18. ¿.Hasta qué punto considera que a los } \\
\text { profesores nos es legitimo utilizar nuestra } \\
\text { condición de docentes para transmitir } \\
\text { valores relacionados con la sostenibilidad? }\end{array}$ & no respuestas & $\%$ \\
\hline $\begin{array}{l}\text { 1. Creo que no es ético aprovechar nuestra } \\
\text { posición como docentes para transmitir nuestros } \\
\text { valores a los estudiantes }\end{array}$ & 2 & 3,8 \\
\hline $\begin{array}{l}\text { 2. Asuntos como la sostenibilidad, tienen tanta } \\
\text { relevancia que, en cuanto están relacionados con } \\
\text { valors morales, debemos ser activos, no pasivos, } \\
\text { cuando los tratamos en las aulas (violencia, } \\
\text { respeto, solidaridad, cuidado del medio ambiente, } \\
\text {...) }\end{array}$ & 47 & 90,4 \\
\hline 3. Otra afirmación diferente de las anteriores & 3 & 5,8 \\
\hline Total general & 52 & 100,0 \\
\hline Media & 1,96 & \\
\hline Índice $\mathrm{Pi}$ & 0,96 & \\
\hline
\end{tabular}

Solamente el 3,8\% encuentra que no es ético aprovechar su posición como docentes para transmitir sus valores a los estudiantes. La mayor parte de los docentes, el 90,4\%, considera que a los profesores les es legítimo utilizar su condición de docentes para adoptar una actitud activa en el tratamiento de valores relacionados con la sostenibilidad en el aula.

\section{Modificación de contenidos conceptuales, procedimentales y actitudinales:}

En las siguientes cuestiones se establece la relación con los contenidos conceptuales, procedimentales y actitudinales.

Para modificar los contenidos conceptuales, los profesores tuvieron que elegir una de entre las opciones que se les ofrecían. La respuesta mayoritaria es la que se inclina por relacionar algunos o todos los temas del programa con cuestiones medioambientales o de sostenibilidad (46'2\%). Las otras dos opciones (definiendo competencias cognitivas para la sostenibilidad dentro del programa de la asignatura o promoviendo en el aula debates sobre la sostenibilidad) merecen el favor de un $15,4 \%$ de los profesores entrevistados, respectivamente. Dos profesores que se decidieron a especificar otras opciones proponen: «No los modificaría»; «Ya lo estoy haciendo impregnando el currículum y con un tema específico».

Para modificar los contenidos procedimentales, se ofreció a los profesores la posibilidad de elegir una entre las opciones y el $42,3 \%$ de los profesores opta por pedir a los alumnos la realización de ejercicios prácticos relacionados con el medio ambiente. A considerable distancia, el 23,1\% se inclina por la primera propuesta que es «definiendo competencias metodológicas para la sostenibilidad dentro del programa de la asignatura». Solo un 9,6\% decide introducir el asunto en el Prácticum como opción preferencial.

Respecto a la modificación de actitudes, los profesores seleccionan las opciones según puede verse en la tabla 3. 
Tabla 3.

Incidencia en la modificación de actitudes del alumnado

\begin{tabular}{|c|c|c|}
\hline $\begin{array}{l}\text { P19.3 ¿Cómo incidiria Vd., principalmente, } \\
\text { en las actitudes de los alumnos? }\end{array}$ & no respuestas & $\%$ \\
\hline $\begin{array}{l}\text { 1. No es procedente realizar acciones } \\
\text { encaminadas a incidir sobre las actitudes } \\
\text { personales de nuestros alumnos. Hay que } \\
\text { esperar que ellos las vayan cambiando por su } \\
\text { cuenta, a la vista de lo que han estudiado }\end{array}$ & 2 & 3,8 \\
\hline $\begin{array}{l}\text { 2. Definiendo competencies actitudinales para la } \\
\text { sostenibilidad dentro del programa de la } \\
\text { asignatura }\end{array}$ & 11 & 21,2 \\
\hline $\begin{array}{l}\text { 3. Haciendo ejercicios de juegos de simulación, } \\
\text { estudio de casos, discusión sobre dilemes éticos, } \\
\text {..., en el ámbito de las clases prácticas }\end{array}$ & 20 & 38,5 \\
\hline 4. Otras & 6 & 11,5 \\
\hline 9. NC & 13 & 25,0 \\
\hline Total general & 52 & 100,0 \\
\hline
\end{tabular}

El 38,5\% prefiere la tercera opción, en la que se proponen prácticas concretas. Cabe destacar que el $25 \%$ opta por no sabe o no contesta a esta cuestión.

\section{Cuestiones genéricas sobre la introducción de la sostenibilidad en los currícula (P20 a P26)}

\section{Posibilidad de introducir competencias para la sostenibilidad en las materias}

La respuesta mayoritaria, con el $51,9 \%$ es la segunda, es decir, la que refleja una mejor voluntad y un cierto compromiso de los profesores (figura 5).

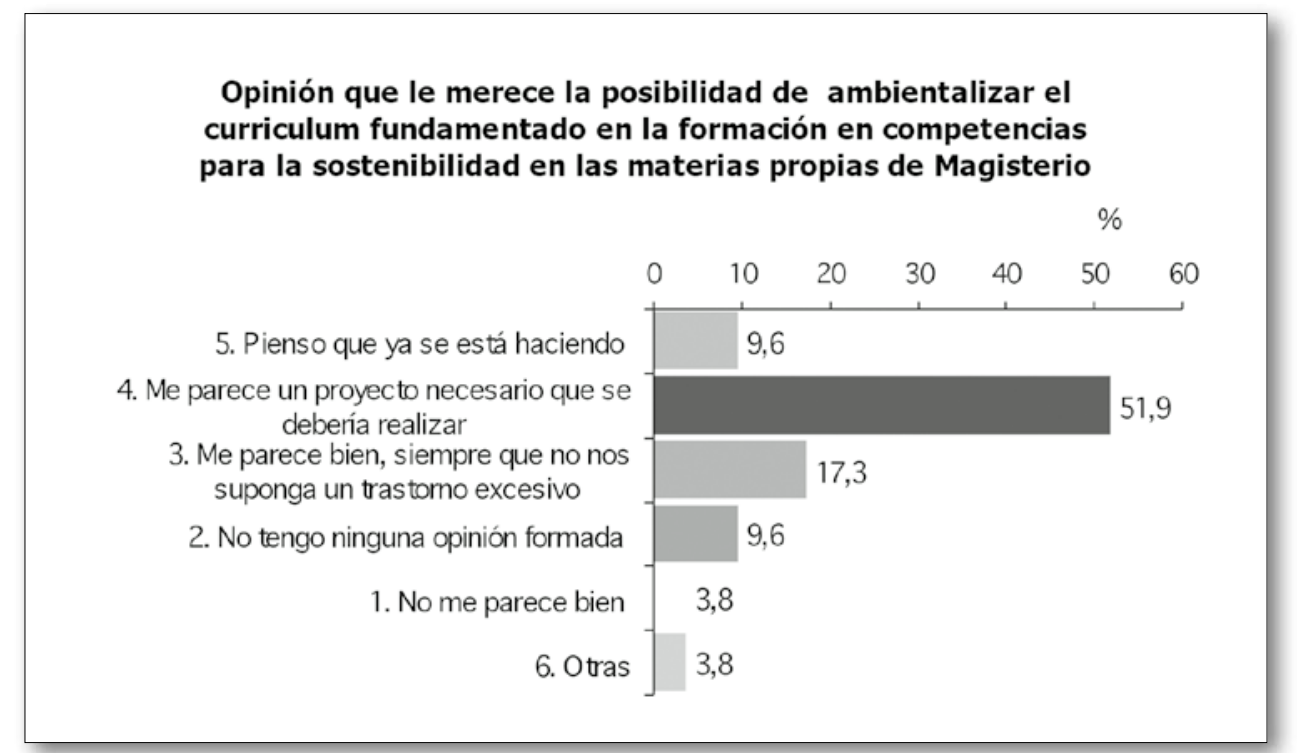

Fig 5. Posibilidad de introducir competencias para la sostenibilidad en las materias 


\section{Desarrollo de competencias emocionales}

Casi el 60\% del profesorado encuestado dice que «conoce y/o aplica métodos, recursos o estrategias para gestionar emociones y sentimientos hacia el medio ambiente y la sostenibilidad, para desarrollar competencias emocionales en los procesos de formación, tanto a nivel individual como grupal». El $40 \%$ dice no conocerlos o aplicarlos.

\section{Fórmula para introducir la sostenibilidad en la docencia}

Para decidir cuál es la fórmula, o sistema, que considerarían más efectiva para introducir la sostenibilidad curricular en los estudios de Magisterio, el profesorado encuestado opta, preferentemente, un $36,5 \%$, por la creación de un seminario permanente por titulación, siendo que en Magisterio conviven dos titulaciones. A no mucha distancia se sitúa el $28,8 \%$, que se inclina por trabajar en ello en el ámbito departamental. Un $21,2 \%$ prefiere circunscribirse al trabajo individual. Un $9,6 \%$ prefiere otras fórmulas. Se observa que la opinión del profesorado está muy diversificada.

\section{Incidencia profesional de los maestros en asuntos relacionados con el medio ambiente y el desarro-} llo sostenible cuando se insertan en el mercado laboral

Se preguntaba al profesorado, específicamente, si los titulados en educación infantil y primaria pueden incidir profesionalmente en asuntos relacionados con el medio ambiente y el desarrollo sostenible cuando se inserten en el mercado laboral.

El 82,7\% de la muestra afirma que los profesores de educación infantil o primaria pueden incidir bastante o mucho en cuestiones relacionadas con el medio ambiente y el desarrollo sostenible, y lo más destacable es que el 51,9\% califican esa incidencia como mucha.

Era importante saber cómo entendían que se podía producir ese efecto sobre el entorno, quienes respondieran que como mínimo puede haber algo de influencia. Para ello se efectuó la siguiente pregunta sobre en qué áreas o aspectos se puede producir esta incidencia profesional del alumnado y cuyos resultados pueden verse en la figura 6 .

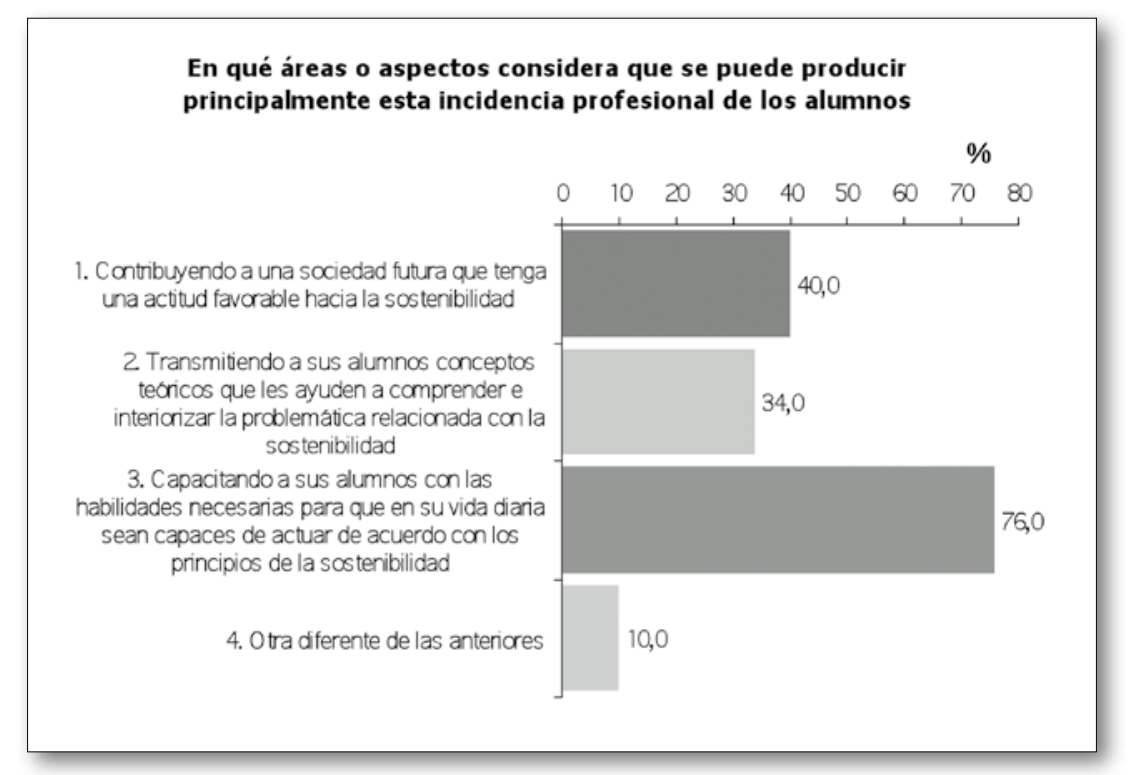

Fig. 6. Áreas o aspectos en los que los maestros pueden incidir profesionalmente 
El 76\% está de acuerdo con la tercera respuesta, con una visión indudable, en profundidad, de los efectos de la enseñanza sobre los niños en sus primeros años de vida y la proyección que los conocimientos adquiridos por ellos en esa época pueden alcanzar en su vida adulta.

\section{Programas o experiencias relacionados con la educación para el Desarrollo Sostenible}

Un 46,2\% dice conocer algún programa o experiencia relacionados con la educación para el desarrollo sostenible dentro de su área de docencia o investigación. El 28,8\% dice que no conoce pero estaría interesado en tener información para reorientar la docencia. Un 23,1\% no conoce ningún programa ni experiencia al respecto.

\section{Predisposición a participar en actividades de formación}

El 30,8\% manifiesta su buena disposición a participar en cursos, encuestas, seminarios, jornadas, etc., dirigidos a favorecer la sostenibilidad curricular en la universidad. El 9,6\% lo niega, lo que confirma el aproximadamente $10 \%$ citado en varios lugares en líneas anteriores que parece resistente a la temática. El $57,7 \%$ se acoge a la respuesta que facilitaba eludir todo compromiso, con un 'depende' absolutamente inconcreto, que podía luego concretarse, o no, en una cuestión abierta. A la pregunta abierta han respondido un $42,3 \%$ y aducen que depende del tiempo que tengan, de los horarios, de la credibilidad de la iniciativa, de la temporalización de los cursos, de lo relacionado que esté con su asignatura, de si está en su línea de investigación.

Por último, puntualizar que no se ha hecho mención a diferencias marcadas entre profesores y profesoras en las variables analizadas. Ello se debe a que, habiendo realizado todos los cruces pertinentes, no se han hallado diferencias suficientemente significativas.

\section{DISCUSIÓN Y CONCLUSIONES}

Respecto a la pregunta sobre la problemática socio-ambiental, más del $60 \%$ del profesorado entrevistado afirma que contribuir al desarrollo sostenible es inherente a una actitud profesional respetuosa con el medio, que es la opción más comprometida de entre las posibles respuestas. Comparando este resultado con el obtenido para esta misma pregunta en una encuesta anterior, realizada a todo el profesorado de la Universitat de Valencia (Ull et al, 2010b), vemos que en el caso del profesorado de Magisterio encuestado hay un mayor compromiso ya que esta respuesta obtuvo, en el caso de la muestra del total de profesores, un 48,9\%. Así, aun siendo en las dos muestras mayoritaria la misma respuesta, existen diferencias significativas. Este dato supone, a priori, un factor positivo para la aplicación y desarrollo de las competencias para la sostenibilidad en la docencia de las titulaciones de Magisterio pues se cuenta con un profesorado que en su mayoría dice estar comprometido con el medio ambiente.

Más de la mitad del profesorado encuestado opina que la introducción de contenidos y enfoques relacionados con la sostenibilidad en su ámbito de docencia puede ser una medida apropiada y, además, afirma que ya lo está haciendo. Es un porcentaje alto y que se corresponde con las respuestas referidas a la introducción de los temas que potencia la UNESCO dentro de la Década de la Educación para el DS.

De igual manera hay una correspondencia notable con el hecho de que la mayoría del profesorado cree que es factible introducir contenidos y enfoques relacionados con la sostenibilidad en las asignaturas que imparten o coordinan en las titulaciones de maestro, pero son de nuevo muy críticos cuando el profesorado encuestado opina mayoritariamente que «aún hay mucho por hacer, aunque en 
los planes de estudios actuales se incluyen acciones específicas de formación (sensibilización, técnicas, habilidades...), que preparan a los futuros graduados de las titulaciones de Magisterio para afrontar los problemas relacionados con la sostenibilidad en el ámbito de sus competencias profesionales como futuros maestros».

La interacción entre profesorado que imparte la misma asignatura en las titulaciones de grado e incluso la interacción con otros/as profesores/as es una variable importante que se debe tener en cuenta en el desarrollo de un modelo de formación por competencias; dicha interacción facilita la realización de acciones tendentes a desarrollar las competencias generales y específicas de la titulación que se tienen que conseguir para graduarse. En el caso del profesorado encuestado el 78,8\% manifiesta tener establecidas dichas interacciones, aspecto muy positivo para el objetivo que hay que alcanzar.

Se constata que los profesores reconocen que las acciones que realizan en el aula tienen una repercusión exterior a las clases; por tanto, es un aprendizaje del cual se aplica y genera una transferencia. Esta ha sido la hipótesis básica de la Teoría de la Educación en su versión clásica, ya que desde la educación siempre se ha tenido la convicción de que lo que el alumnado aprende en un contexto determinado (por ejemplo el aula) lo va a transferir y a aplicar a las diferentes situaciones que se le puedan presentar en su vida. Esta hipótesis ha sido confirmada posteriormente, al incluir en la explicación del proceso de transferencia cuestiones relacionadas no solo con el qué se aprende (conocimientos), sino también con el cómo se aprende (procedimientos, métodos, estrategias), de quién se aprende, dónde se aprende, para qué se aprende (objetivos)... Parece obvio que los procesos de enseñanza y aprendizaje que realiza el profesorado encuestado están en esta línea, lo que garantiza en mayor medida un aprendizaje práctico y de competencias.

Cuando se les pregunta si trabajan desde su asignatura los valores de respeto, tolerancia, equidad, etc., como componentes de la sostenibilidad, casi la totalidad responde afirmativamente. Y, respecto a las formas de trabajar estos aspectos, un número significativo de estos declaran trabajar estos aspectos por convicción y formación; sin embargo, todavía hay un porcentaje de los que trabajan estos valores que los vinculan con aspectos externos, como la exigencia de la propia asignatura o cuestiones de actualidad.

Nos encontramos que casi todo el profesorado está de acuerdo con tener una actitud activa en el tratamiento en el aula de los valores relacionados con la sostenibilidad. Se puede establecer, pues, que los docentes entienden que, al menos en lo que atañe a la sostenibilidad, su trabajo no es neutro y está muy vinculado con los aspectos éticos y de escala de valores. Las tendencias en la investigación sobre valores en la educación están abogando no por enseñar valores sino por ayudar a clarificar los valores a adoptar por parte de los alumnos y también es así en educación ambiental, tal y como discute Mayer (1998).

Más del 75\% de los profesores encuestados creen necesario y/o posible la introducción de competencias para la sostenibilidad en las materias propias de Magisterio. Este es un dato positivo y un estímulo para seguir con procesos de introducción de la sostenibilidad de la docencia, ya que ahora sabemos que se puede contar con lo imprescindible, que es el profesorado, de una forma mayoritaria.

En síntesis planteamos algunas consideraciones de carácter general, aplicables a todo el profesorado y también en concreto al profesorado de Magisterio, a modo de conclusiones para avanzar:

- El conocimiento de la sostenibilidad por parte de los docentes es aun relativamente escaso; y todavía hay más desconocimiento acerca de cómo aplicarlo en sus especialidades o asignaturas, lo que es una barrera para incorporarla en su docencia. Así pues, haría falta disponer de libros de texto o artículos especializados de referencia que relacionen la problemática ambiental, la sociedad y la economía. Y sobre todo orientaciones y recursos que sirvan de apoyo para adaptar tales conocimientos a las asignaturas. 
- Se detecta como barrera importante la falta de tiempo que el profesorado aduce para incorporar actividades relacionadas con la sostenibilidad en sus materias, con el temor a una pérdida de contenidos y tiempo para la comprensión de los aspectos más específicos de su asignatura.

- No obstante las reticencias de parte del profesorado parecen ser de tres tipos:

a) falta de concienciación del problema (no ven la necesidad de cambio hacia la sostenibilidad);

b) falta de implicación (creen que hace falta el cambio pero no ven relación entre las asignaturas que imparten y la sostenibilidad);

c) falta de capacitación (hay profesores que quieren el cambio y quieren hacerlo pero no saben cómo hacerlo, son los que reclaman materiales y formación). Todo ello viene a confirmar y ampliar lo dicho ya por otros autores sobre las barreras para la educación para el desarrollo sostenible (Holmberg y Samuelson, 2006; Lozano, 2006, GranadosSánchez et al., 2012).

- Para propiciar la superación de las barreras es importante que los docentes puedan contar con una Guía de sostenibilidad en la docencia que les permita desarrollar las competencias para la sostenibilidad e introducirla en las guías docentes de las distintas asignaturas, ya que todo lo que no se planifica no se hace. Y es fundamental, tal y como indican Pramling-Samuelsson y Kaga (2010), que se introduzcan esas competencias para que se transforme el modelo cultural hacia la sostenibilidad en la educación desde la primera infancia.

\section{BIBLIOGRAFÍA}

Aznar Minguet, P. (2006). El reto educativo de la sostenibilidad en el marco europeo de la educación superior. En A. Escolano Benito, Cambio educativo y cultura de la sostenibilidad. Madrid: Biblioteca Nueva.

Aznar Minguet, P. y Ull, M. A. (2009). La formación de competencias básicas para el desarrollo sostenible: el papel de la Universidad. Revista de Educación no extraordinario 2009, pp. 219-237.

Aznar, P., Martínez-Agut, M. P., Palacios, B., Piñero, A. y Ull, M. A. (2007). Ambientalización curricular en la Universitat de València. (ACUVEG 2005). I.S.B.N.: 978-84-935288-1-2.

Aznar, P., Martínez-Agut, M. P., Palacios, B., Piñero, A. y Ull, M. A. (2009). Cuestionario de ambientalización curricular para profesores de las titulaciones de Educación Infantil y Educación Primaria (profesores, 2008). I.S.B.N.: 978-84-92690-29-9.

Aznar Minguet, P., Martinez-Agut, M., Palacios, B., Piñero, A. y Ull, M. A. (2011). Introducing sustainability into university curricula: an indicator and baseline survey of the views of university teachers at the University of Valencia. Environmental Education Research, 17 (2), pp. 145- 166. http://dx.doi.org/10.1080/13504622.2010.502590

Aznar Minguet,P., Ull M.A., Piñero,A y Martínez-Agut, M.P. (2014). Competencias básicas para la sostenibilidad.Un análisis del diálogo disciplinar. Bordón 66(2), pp.13-27

Barrón, A., Navarrete, A. y Ferrer-Balas, D. (2010). Sostenibilización curricular en las universidades españolas. ¿ha llegado la hora de actuar? En Rev. Eureka Enseñanza y Divulgación de las Ciencias, 7, No Extraordinario: La sostenibilidad en la universidad, pp. 388-399.

Barth, M., Godemann, J., Rieckmann, M. y Stoltenberg. U. (2007). Developing Key Competencies for Sustainable development in Higher Education. International Journal of Sustainability in Higher Education, Vol. 8 (4), pp. 416-430.

http://dx.doi.org/10.1108/14676370710823582 
Bonil, J., Calafell, G., Márquez, C. y Pujol, R.M. (2004). La integración del paradigma de la complejidad en la formación científica como vía de acceso a la ambientalización curricular: las preguntas mediadoras y el diálogo disciplinar. En A.M. Geli y M. Junyent (eds.). Ambientalización Curricular de los Estudios Superiores. Girona: Universitat de Girona-Red ACES.

Elizalde, A. (2009). ¿Qué desarrollo puede llamarse sostenible en el siglo XXI? Revista de Educación, $\mathrm{n}^{\circ}$ extraordinario 2009 , pp. 53-75.

Geli, A. Ma (2002). Universidad, sostenibilidad y ambientalización curricular. En Ambientalización Curricular en los Estudios Superiores. I. Aspectos Ambientales de las Universidades. Arbat. E. y Geli, A.M. eds. Universitat de Girona: Servei de Publicacions /Red ACES.

Granados-Sánchez, J., Wals, A. E. J., Ferrer Balas, D., Waas, T., Imaz, M., Nortier, S., Svanström, M., Van't Land, H. y Arriaga, G. (2012). Sustainability in Higher Education: moving from understanding to action, breaking barriers for transformation. En Higher Education in the World 4. GUNI Series son the Social Commitment of Universities 4, pp. 193-207. New York: Palmgrave Macmillan.

Holmberg, J. y Samuelson, B. (eds.) (2006). Drivers and barriers for implementing sustainable development in higher education. Paris: UNESCO. http://unesdoc.unesco.org/images/0014/001484/148466E.pdf.

Junyent, M., Bonil, J. y Calafell, G. (2011).Evaluar la ambientalización curricular de los estudios superiores: un análisis de la red Edusost, Ensino Em Re-Vista, 18 (2), pp. 323-340.

LozANo, R. (2006). Incorporation and institutionalization of sustainable development into universities: Breaking through barriers to change. Journal of Cleaner Production 14 (9-11), pp. 787-96. http://dx.doi.org/10.1016/j.jclepro.2005.12.010

Martinez-Agut, M. P., Aznar Minguet, P., Ull, M. A. y Piñero, A. (2007). Promoción de la sostenibilidad en los currícula de la enseńanza superior desde el punto de vista del profesorado: un modelo de formación por competencias. En Educatio Siglo XXI, 25 (Educación y sostenibilidad), pp. 187-208. Universidad de Murcia.

Mayer, M. (1998). Educación Ambiental: de la acción a la investigación. En Enseñanza de las Ciencias, 16 (2) pp. 217-231.

Miranda, M. J. y Regí, C. (2006). Educación para la sostenibilidad: un compromiso con el futuro, en La sostenibilidad, un compromiso de la escuela. En I. Askasibar et al., pp. 53-64. Claves para la Innovación educativa no 37. Barcelona: Graó.

Morin, E (2011). La vía. Barcelona: Paidos.

OrR, D.W. (2010). ¿Para qué sirve ahora la educación superior? En: La situación del mundo 2010: Cambio Cultural: del consumismo hacia la sostenibilidad, pp. 155-167 Barcelona: The WorldWatch Institut. Icaria Editorial.

Piñero, A., Ull, M. A., Martínez, M.P. y Aznar, P. (2006). La Formación para la Sostenibilidad: el Indicador Pi como medida de la Ambientalización Curricular. Ed. I International Conference on Sustainability Measurement and Modelling (ICSMM 2006). Disponible en: <congress.cimne.upc.es/ icsmm06/esp/Proceedings.asp>.

Pramling Samuelsson, I. y Kaga, Y. (2010). La educación en la primera infancia para transformar el modelo cultural hacia la sostenibilidad. En: La situación del mundo 2010: Cambio Cultural: del consumismo hacia la sostenibilidad, pp. 125-132, Barcelona: The WorldWatch Institut. Icaria Editorial.

Pujol, R. M. (2006). Construir una escuela que eduque para el desarrollo sostenible. En La sostenibilidad, un compromiso de la escuela. I. Askasibar et al., pp. 27-36. Claves para la Innovación educativa no 37. Barcelona: Graó.

SAuvé, L. (2010). Educación Científica y Educación Ambiental: un cruce fecundo. En Enseñanza de las Ciencias, 28 (1), pp. 5-18. 
STERLING, S. (2005). Higher education, sustainability, and the role of systemic learning. En P.B. Blaze Corcoran y A.E.J. Wals (eds). Higher Education and the Challenge of Sustainability: Problematics, Promise and Practice, pp. 47-70. Dordretch: Kluwer Academic Press

Toledo, V. M. (1999). "Universidad y Sostenibilidad. Cinco tesis y una propuesta para el nuevo milenio». Ponencia Marco El papel de las universidades. III Congreso de Universidades por el Desarrollo Sostenible y el Medio Ambiente (1999). Universidad de Valencia (CD-ROM).

Ull, M. A., Martínez-Agut, M. P., Piñero, A. y Aznar Minguet, P. (2010a). Análisis de la introducción de la sostenibilidad en la enseñanza superior en Europa: compromisos institucionales y propuestas curriculares. Revista Eureka sobre Enseñanza y Divulgación de las Ciencias. Número extraordinario 2010, pp. 413-432.

Ull, M. A., Martínez Agut, M.P., Aznar, P. y Piñero, A. (2010b). Conocimientos y actitudes del profesorado universitario sobre problemas ambientales. Revista Enseñanza de las Ciencias, 28(3), pp. 433-446.

UNESCO (2005a). Decenio de las Naciones Unidas para el desarrollo sostenible 2004-2015: Plan de aplicación internacional. Proyecto. París: UNESCO.

UNESCO (2005b). Directrices y recomendaciones encaminadas a reorientar la formación de docentes para abordar el tema de la sostenibilidad. La educación para el Desarrollo sostenible en la práctica. Documento Técnico no 2. París: UNESCO.

Vega Marcote, P. y Álvarez SuÁrez, P (2011): La agenda 21 y la huella ecológica como instrumentos para lograr una universidad sostenible, en Enseñanza de las Ciencias, 29(2), pp. 207-220

Vilches, A. y Gil Pérez, D. (2009). Una situación de emergencia planetaria, a la que debemos y «podemos» hacer frente. Revista de Educación, número extraordinario 2009, pp. 101-122. 


\title{
Preconcepcions and attitudes of teachers of Teaching School about incorporated sustainability competences in their teaching
}

\author{
M. Ángeles Ull, Albert Piñero, M. Pilar Martínez-Agut, Pilar Aznar Minguet \\ Universidad de Valencia \\ angels.ull@uv.es, albert.pinero@uv.es,mdelpi@uv.es, pilar.aznar@uv.es
}

The present study aims to specify basic competences adopting sustainability criteria to reformulate and guide curricular programs to apply them in different educational practices. From this perspective, an education for sustainable development in the 21 st century can help society to deal with different problems; first and foremost those relating to water, energy, climate change, mitigation of risk and disasters, loss of biodiversity, food crisis, threats to the health, social vulnerability or insecurity.

The present work is framed within a broader research project on the introduction of competences for sustainability in the curriculum of Early Childhood and Primary Education Degree. A seminar of disciplinary dialogue has been held with teachers of the current Education Degree at the University of Valencia, whose results will be collected in another publication (Aznar et al, 2013). Prior to the aforementioned seminar and to prepare for this one, two surveys were conducted with teachers and training teachers. The study presented here is the result of the survey for teachers, which allows us to know the starting point for the incorporation of competences for sustainability in their teaching.

The specific objectives are: to analyse the normative context of sustainability training in new programmes of studies for the Early Childhood Education Teachers Degree and Primary Education Degree and to review references on the sustainability-related competences included in the new syllabus. To this aim, we begin studying the teachers' preconceptions and attitudes towards the introduction of sustainability into the mentioned degrees.

To know the reality of the target study, a questionnaire has been used with early childhood and primary education teachers. The questionnaire has been devised to learn about early childhood and primary education teachers' preconceptions and attitudes. It contains 31 variables relating to the theme that have been classified into 4 categories: Identification (Degrees taught, Assignment department, Professional status, Age, Gender); Perception; Interpretation and Performance (Attitudes towards sustainability, Willingness to include sustainability into teaching and such).

The questions in the questionnaire asked teachers about: their opinions on environmental problems, (Q1), training received at university in the light of sustainability (Q2), updating knowledge (Q3), sustainability in teaching (Q4, Q5), course subjects and actions to be carried out to promote the sustainability culture (Q6 to Q11), actions with students (Q12 to Q19) and generic questions about introducing sustainability into curricula (Q20 to Q26).

In order to present this paper for international broadcasting, we selected the more general issues: Environmental problems (Q1); Sustainability in teaching (Q4); Themes and actions to carry out to promote the sustainability culture (Q6); Introduction of contents and approaches relating to sustainability into the course subjects (Q8), Specific training actions (Q9) and Interaction between the teachers (Q11). 